Proceedings of the 2011 Winter Simulation Conference

S. Jain, R.R. Creasey, J. Himmelspach, K.P. White, and M. Fu, eds.

\title{
A SIMULATION-BASED MODELING FRAMEWORK TO DEAL WITH CLINICAL PATHWAYS
}

\author{
Yasar A. Ozcan \\ Virginia Commonwealth University \\ Department of Health Administration \\ 1008 East Clay Street \\ Richmond, VA 23298, USA
}

\author{
Elena Tànfani \\ Angela Testi \\ University of Genova \\ Department of Economics and \\ Quantitative Methods (DIEM) \\ Via Vivaldi 5, 16126, Genova, ITALY
}

\begin{abstract}
In this paper we focus our attention on the analysis and management of Clinical Pathways (CPs) in health care systems. From an operational point of view, the CP is "the path" followed by a patient with a given pathology through the health-care system. We start by a global vision and propose a modeling framework based on a discrete event simulation model to identify the critical activities and scarce resources that represent the process bottlenecks both from a patient-centered and facility-centered point of view. Moreover, we face the challenging problem of integrating simulation and optimization in order to put together the capability of the simulation in the scenario analysis ("what-if" analysis) and in describing the dynamics of the system considered and the decisional strength of the optimization, i.e., the "what-best" analysis. The framework is applied to a case study for the thyroid surgical treatment.
\end{abstract}

\section{INTRODUCTION}

Clinical Pathways (CPs), also known as Integrated Care Pathways, Multidisciplinary Pathways of Care, Pathways of Care, Care Maps, Collaborative Care Pathways, were first introduced in the early 90s in UK and USA; then their application spread throughout the western world (Zander 2002). CPs are defined as "health-care structured multidisciplinary plans that describe spatial and temporal sequences of activities to be performed, based on the scientific and technical knowledge and the organizational, professional and technological available resources" (De Blaser et al. 2006). They can be seen as algorithms, described by flow-charts, detailing the set of decisions and treatments to be given to the patient, with a logic based on sequential phases. From an operational point of view, the CP is therefore "the path" followed by an ill person through the health-care system. This path can be analyzed at a single level of care (e.g., inpatient care, outpatient care, home care, primary care) or globally, taking into account every level of health-care from education and prevention, to diagnosis, treatment and recovery.

The crucial characteristics of CPs is that they are patient-centered and not facility-centered. They can, therefore, be considered as an operational tool in the clinical treatment of diseases, from a patient-focused point of view (Panella, Marchisio, and Stanislao 2003). They are especially tailored to stimulate continuity and coordination among the treatments given to the patient through different disciplines and clinical environments. The ultimate aim of CPs is to reduce variability in outcomes and costs by: a) standardizing treatment and care protocols for specific conditions; b) coordinating the patient journey through the entire care process. In practice, the second task is difficult to be implemented and, as far as we know, the literature is usually focused on a limited part of the care process (mainly the hospital phase). In many health care systems, extending the analysis to further phases and facilities is almost impracticable due to the lack 
of administrative data linking the different levels of care that could allow monitoring the patient path continuously.

The complexity of the problem requires an integrated approach where different economic and quantitative tools as well as simulation and optimization techniques can find their place (Brandeau, Sainford, and Pierskalla 2004; Ozcan 2009; Vissers and Beech 2005). Even if many experiences for specific diseases can be found in the medical literature, at least to the writers knowledge, there is no general recommendation or technique that can be used as a decision support tool in the general case. Instead the OR\&MS literature is very poor. Some authors propose discrete-event simulation models aimed at investigating the global flow of patients belonging to different paths, focusing only on the hospital phase or ambulatory phase (Cardoen and Demeulemeester 2008; Swisher et al. 2001). Maliapen and Dangerfield (2010) propose a system dynamics-based simulation approach to examine the development of clinical pathways in a hospital in Australia, while Cote and Stein (2007) develop a semi-Markovian queuing model for modeling the flow of a single patient during a treatment given in an ambulatory structure.

The aim of this paper is to develop a modeling framework aimed at analyzing the pathway of patients with common acute diseases, highlighting not only variability but also critical points and bottlenecks, and identifying the organizational model of care, thus allowing the proper sizing and optimal management of resources. Instead of using a hypothetical example with a specific disease as an illustration, we present a case study for the thyroid surgical treatment using the data collection process at the Department of Surgery of a public hospital sited in Genova (Italy). Note that thyroid is a good example for clinical pathways because it fits most of the selection criteria suggested by Zander and Bower (1987), such as: 1) prevalent pathology within the care setting, 2) pathology with a significant risk for patients, 3) predictable clinical course, 4) pathology well defined permitting a homogeneous care, 5) existence of recommendations of good practices or experts opinions.

\section{MODELING FRAMEWORK}

The framework herein proposed is schematically described in Figure 1. The novelty of the proposal regards both methods as well as expected results. As far as methods are concerned, it can be noted that different modeling techniques (project management, simulation and optimization models) are integrated in the framework giving different informative outputs. This approach allows to assess and optimize the CP performance in terms of a set of indicators taking into account both the patient and the facility point of view. In the following the main steps of the framework are briefly described.

Step1. In order to identify the nature of the flow process, and to create a functional clinical pathway the first step is identifying the fundamental activities that make up the process of health care delivery as an integrated project with many tasks to be executed (Breakdown Activity Structure). This requires to create, with the clinicians and personnel involved in the care process, a simple flow chart that must effectively represents the relevant activities, that characterize the clinical path of patients with a given pathology, as well as the causality and precedence relations among them. These information are used to identify the CP and implement a project management study by following four steps: 1) identification of activities, 2) identification of relationships among activities, 3) identification of time requirements for the activities (deterministic and probabilistic), and 4) identification of the path(s) for care delivery and its duration (Ozcan 2009). Using the classical Critical Path Method (CPM) and Program Evaluation and Review Technique (PERT) it is possible to estimate the project duration, identify the activities most critical to its ontime completion, and calculate how long any activity can be delayed without delaying the project.

We argue that a project management approach helps to conceptualize the path and synthesize how each patient goes through the treatment process. However, there are two main drawbacks: i) solutions are generated assuming infinite resource capacity; ii) waiting times among activities are not considered. 


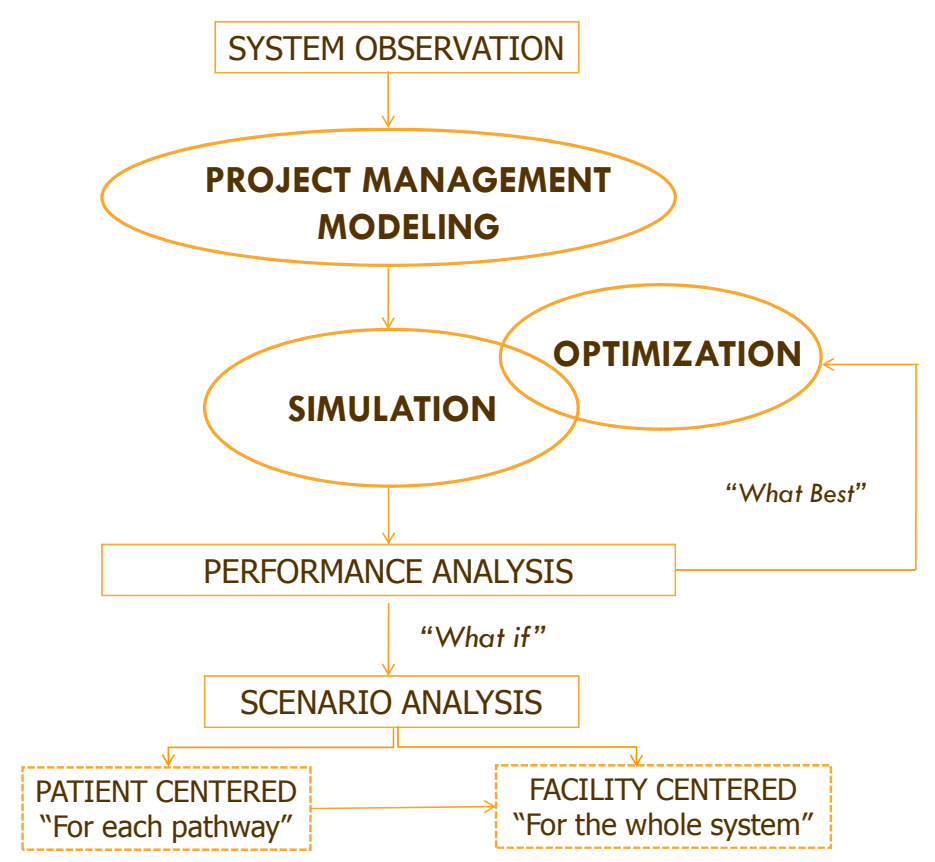

Figure 1: Simple view of the modeling framework

Step 2. In order to take into account the variability and stochastic characteristics of the system as well as the availability of the resources involved in the CP, the use of a simulation model is the more natural and obvious choice. The main output is twofold. First, simulation allows to assess the performance of the system by means of a complete set of indicators. The performance analysis must take into account the patient point of view (average waiting time, average CP length, variability with respect to the clinical guidelines), as well as hospital point of view (throughput, referral and elective waiting list length, under or over utilization of operating rooms, bed occupancy rate and so on). This means from an economic perspective to close the model integrating the demand and the supply side. Secondly, the simulation model can allow us to identify the "scarce" resources that represent the process bottlenecks. Moreover, by the "what if" analysis it is possible to evaluate the impact of organizational changes consisting mainly of rearrangement or capacity increase of the existing resources, learn how the system responds to changes in assumptions, test modifications and assess the effects and process improvements in advance.

Step 3. The third step uses optimization methods to deal with the main CP criticalities stemming from Step 1 and 2. When the system performance is not satisfactory, that is when the gap between real and standard value of some indicators is greater than an objective predetermined value (for instance CP length and waiting time longer than the a priori fixed guaranteed one), some strategies could be detected by means of ad hoc optimization models. For that purpose the DES model must be designed to interact with some optimization models at predetermined instants of the simulation run. After individuating the "what best" solution for a given problem/task, the simulation model allows to validate the optimized solutions with respect to the whole system.

In addition, we face the challenging problem of integrating simulation and optimization ( $\mathrm{Fu} 2002 ; \mathrm{Fu}$, Glover, and April 2005) in order to put together the capability of the simulation in the scenario analysis ("what-if" analysis) and in describing the dynamics of the system and the decisional capability of the optimization, i.e., the "what-best" analysis. Our purpose is to go over the classical approach, in which simulation is just a tool for measuring the goodness of some optimization solutions, in order to develop an integrated approach in which the optimization represents the decision engine for the simulation project. Integration between simulation and optimization allows to get the best scenario, i.e., the choice of param- 
eter values that allow to optimize the trade-off between patient-centered and facility-centered point performance indicators.

\section{APPLICATION TO THE THYROID SURGICAL TREATMENT CASE STUDY}

The framework uses as operative scenario a specialty of the Department of Surgery in San Martino University Hospital sited in Genova (Italy) that is specialized in the thyroid procedure. The existing collaboration with the specialty surgeons give us the possibility to implement the framework for the thyroid surgical treatment. It seems to be an appropriate example for two reasons. First this procedure fits most of the selection criteria suggested by Zander and Bower (1987) recalled above. Secondly, recent studies suggest that clinical pathways for this procedure have the potential to reduce length of stay and limit variability in care, thereby yielding cost savings (Kulkarni et al. 2011; Ramanujam and Cheah 2005; Soria-Aledo et al. 2008).

\subsection{Implementation of the Framework}

Firstly, we share with the clinicians and other personnel involved in the care process of the thyroid treatment the relevant information in order to construct a representative flow chart for the referred treatment (Ozcan, Tànfani and Testi 2011). The developed flow chart follows the patient across three settings: ambulatory, ward and operating theatre. From entry to exit the patient flow is visualized and decisions and actions are identified (Figure 2).

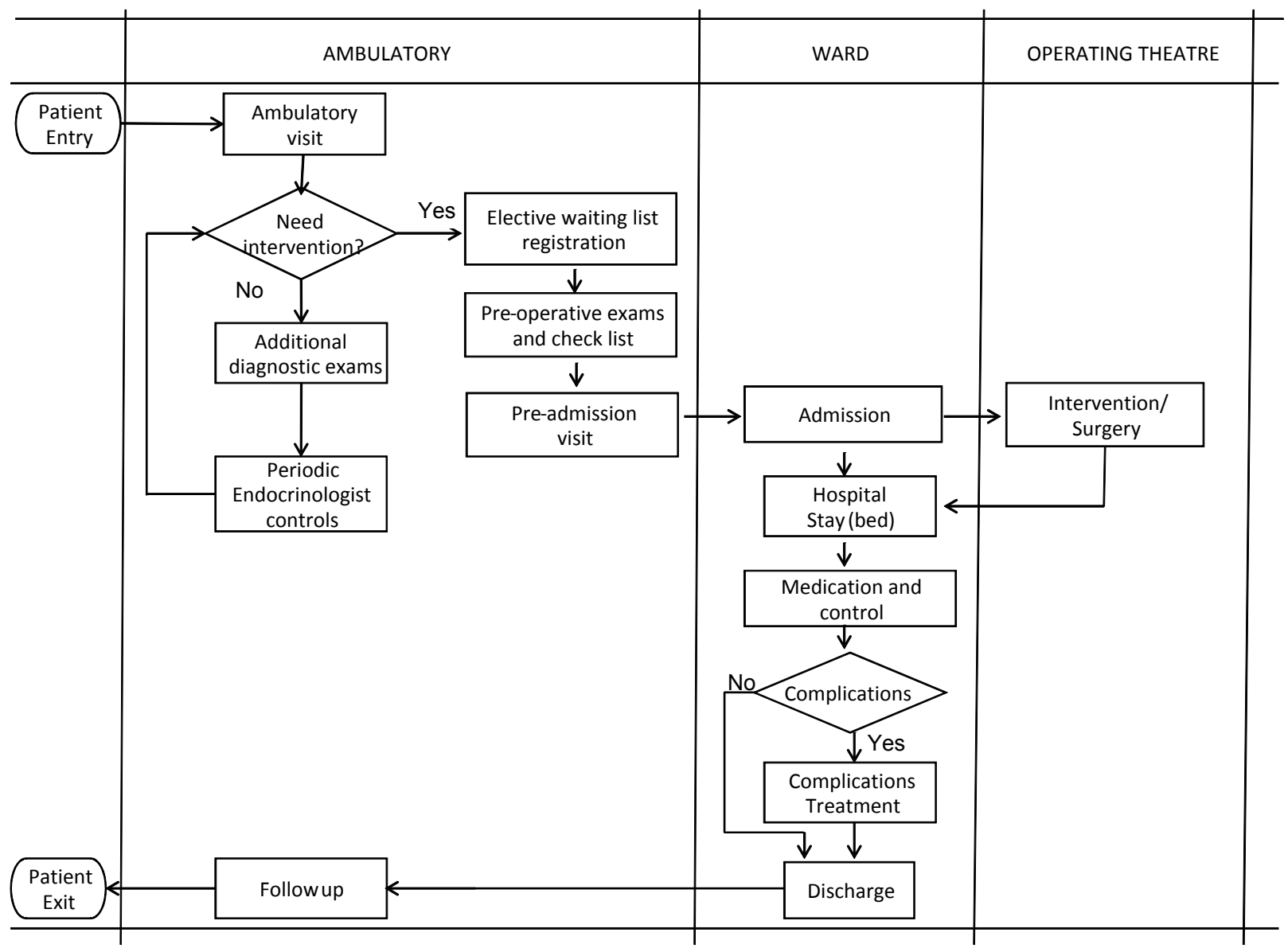

Figure 2: Flowchart of the thyroid pathway 


\section{Ozcan, Tànfani, and Testi}

Note that, the flowchart is now implemented in the current practice and is a crucial tool in periodic internal audits for quality assurance.

Starting from this flowchart, clinical and non-clinical staff in ambulatory, wards and operating theatre were asked to collect the data on the activities. Results of the collection on the time needed to perform each activity on random selected patients are given in Table 1. For each activity, the precedence relationships and the deterministic and probabilistic time durations are reported. Note that the times are all expressed in minutes except for the hospital stay that is expressed in days.

Table 1: Breakdown Activity Structure and times estimation for thyroid treatment

\begin{tabular}{ccccccc}
\hline \multirow{2}{*}{ Activity } & Description & \multicolumn{7}{c}{ Predecessor Deterministic Optimistic } & $\begin{array}{c}\text { Most } \\
\text { Likely }\end{array}$ & Pessimistic \\
\hline A & Visit prenotation & - & 7.5 & 5.0 & 6.0 & 10.0 \\
B & Ambulatory visit & A & 31.5 & 25.0 & 30.0 & 45.0 \\
C & Elective waiting list registration & B & 12.0 & 10.0 & 15.0 & 20.0 \\
D & Pre-operative exams & B & 31.0 & 25.0 & 26.0 & 45.0 \\
E & Check list & D & 28.0 & 25.0 & 25.0 & 35.0 \\
F & Scheduling and planning WL & C.D & 37.0 & 25.0 & 40.0 & 60.0 \\
G & Pre-admission visit & E & 62.5 & 40.0 & 50.0 & 240.0 \\
H & Patient admission & F.G & 32.0 & 25.0 & 30.0 & 45.0 \\
I & Hospital stay (in days) & H & 1.9 & 1.0 & 2.0 & 3.0 \\
L & Intervention/Surgery & I & 96.0 & 40.0 & 90.0 & 316.0 \\
M & Medication and control & L & 38.0 & 30.0 & 34.0 & 60.0 \\
N & Discharge & M & 41.0 & 35.0 & 40.0 & 45.0 \\
\hline
\end{tabular}

These data have been used to estimate the duration of the thyroid surgical treatment under deterministic and probabilistic scenarios as well as to identify the activities most critical and calculate how long any activity can be delayed without delaying the project.

Our preliminary results showed that the deterministic solution suggests 2997.5 minutes to complete treatment under fixed conditions, this translates to approximately 50 hours or two days plus 2 hours. Most of the activities lie on critical path with the exception of activity " $\mathrm{C}$ " (registration for elective wait list) and activity $\mathrm{F}$ (scheduling and planning waiting list). In probabilistic solution the critical path remains same, however, the thyroid treatment mean completion time increases by about 3 hours.

Obviously, the project management approach considers the time needed to complete the thyroid treatment under a "best case" scenario, i.e., under the assumption of infinite resource capacity and hypothesizing that each activity can begin immediately after that all the predecessors have been executed, i.e., the waiting time between activities is not taken into account.

In order to relax the strong assumption that resources are always available to perform each task for each patient, a Discrete Event Simulation (DES) model has been developed using the simulation software Witness (Lanner Group 2010). The DES model overview of the care process of the specialty under study is ported in Figure 3.

The simulation model is designed to include the flow of all patients going across the system, i.e., both the "thyroid" and "other pathologies" patients, since they compete against the same common resources of the specialty, i.e., personnel, ambulatory time, beds, operating theatres and so on. 
Ozcan, Tànfani, and Testi

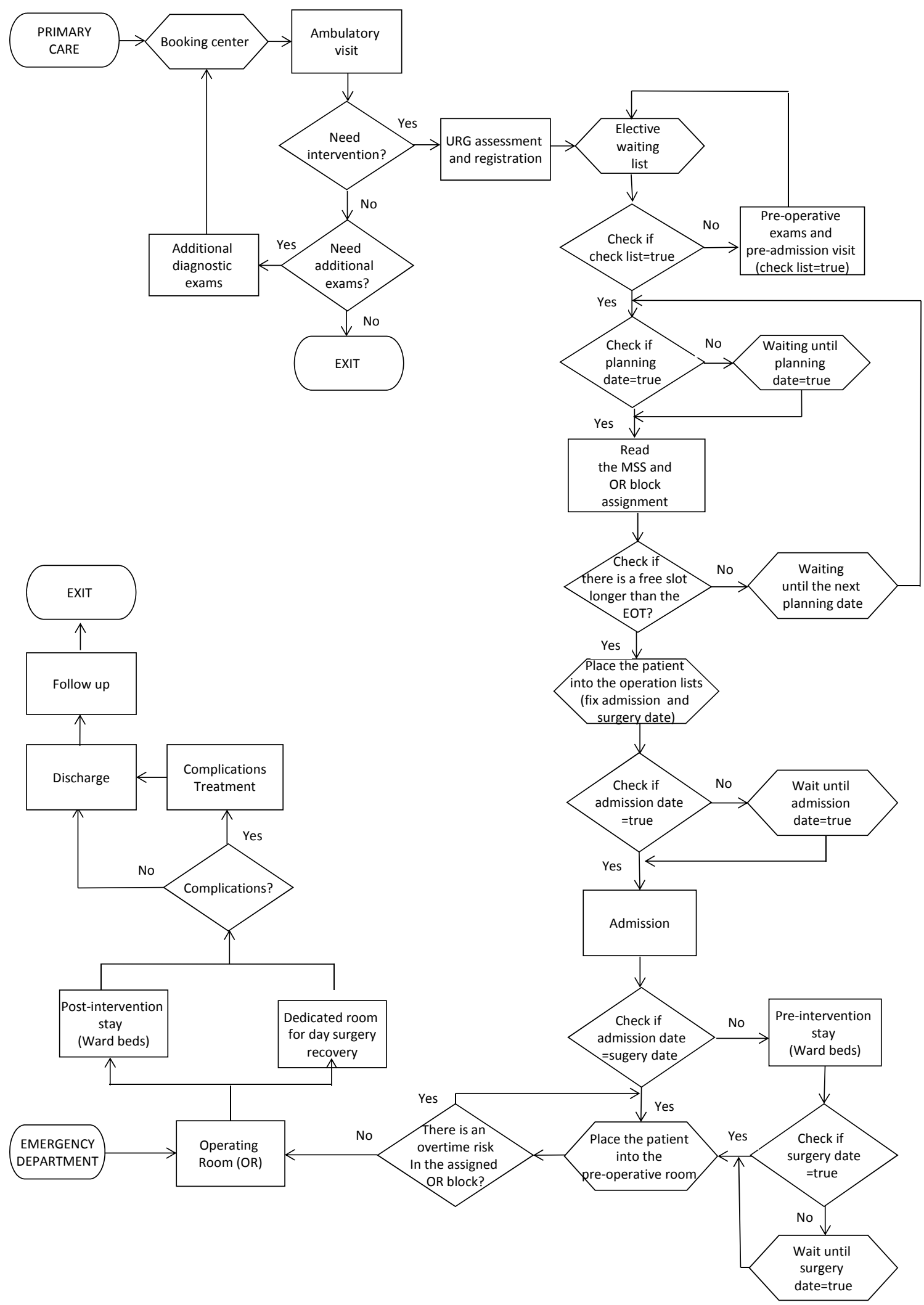

Figure 3: Overview of the DES model representing the patients flow process through the system 


\section{Ozcan, Tànfani, and Testi}

Patients can be admitted into the specialty as elective or emergent. Emergent patients are directly admitted from the Emergency Department to be operated on. Note, however, that this flow does not include the patients coming from the Emergent Department that after few days are dismissed because they do not need intervention or their intervention is postponed and they re-enter as elective patients. Elective patients start the care process by a consultation visit when a clinician decides if they need an intervention. In the latter case additional exams are prescribed and they should be controlled later. On the contrary, if they need an intervention, the surgeon registers them in the elective waiting list and assesses what is the Maximum Time Before Treatment (MTBT) the patient can wait without detrimental consequences on his/her health status. This means assigning the patient to a Urgency Related Group (URG). In the Department under study there are seven urgency categories (A,B,C,D,E,F and G) with a MTBT of 3,15,30,60,90,120 and 180 days, respectively. The elective waiting list is prioritized by means of the percentage of patient MTBT elapsed during the wait that increases every day, depending on the urgency category.

Note that each elective patient has six attributes associated, i.e., URG, updated waiting time, diagnosis, Length Of Stay (LOS), Expected Operating Time (EOT) and Real Operating Time (ROT). The EOT is assessed by the surgeons during the consultation visit, whereas the ROT is the effective processing time of the patient entities and includes the actual duration of the surgical operation, the preparation, the anesthesia and the awakening times together with the room cleaning and set-up times. This represents the total duration of the operation performed in the Operating Rooms (ORs).

At a given planning time the list of patients that are scheduled to be operated on during the next week is decided. The model reads the Master Surgical Schedule (MSS) that is the ORs and block times allocation to the specialty during the next period (usually a week or two weeks). The MSS historical data or the solutions of optimization models (discussed later) can be used to determine the number of OR blocks assigned and their distribution during the week.

Before admitting a patient for a scheduled session (OR block) the model first verifies if the patient has executed the pre-operative exams and anesthesiologist visit following the specialty protocols (see Figure 2); afterwards it checks if the EOT is sufficiently short to be fitted in the unallocated capacity of some OR block. The procedure iteratively continues admitting patients, until the maximum capacity of all blocks are reached and creates the operation lists of all OR blocks at the beginning of the week.

Note that, when the patient is admitted before going into the assigned OR block to be operated on the model must verify if there is an overtime risk, i.e., whether the ROT of the patient is larger than the OR time already available in the assigned OR block time. This rule manages the fact that the effective surgery duration, i.e., the ROT, could be longer with respect to the EOT due to possible complications during the intervention. If there is an overtime risk the patient is shifted to the next scheduled day.

After being operated, patients go into the post-intervention stay beds (ward) for a given period of time depending on the diagnosis, URG and complications. Note that, the so called "Day Surgery", i.e., patients that are dismissed the same day of the intervention, after being operated are placed into a dedicated recovery room where they are kept in observation for signs of any complications until their release.

Data for developing and validating the DES model were collected retrospectively for one year (1 January - 31 December 2009). The main data collected for each patient regards the sex, date of birth, first consultation visit and referral date (necessary to estimate the inter-arrival time), date of admission, surgery date, surgery durations, LOS, EOT and ROT, diagnosis procedure and Diagnosis Related Group (DRG). Note that, to model the specialty activity during the period two sets of administrative data have been collected. The first is related to all elective patients that enter the systems during the period, i.e., join the waiting list (Table 2), while the second collects all the patients (emergent and elective) that have been operated on during the same period (Table 3). In Table 2 the case mix characteristics of the cohort of patients registered on the waiting list is reported. Among the 614 patients registered, 426 have been admitted during the period, while 188 are still waiting at the end of the year and more than $50 \%$ of them have been diagnosed as thyroid patients. For each patient group the average waiting time (in days) is also reported. 
Ozcan, Tànfani, and Testi

Table 2: Case-mix characteristics of the patients entering the system during the period analysis

\begin{tabular}{lccccc}
\hline & \multicolumn{2}{c}{ Admitted } & \multicolumn{2}{c}{ Still waiting } & \multicolumn{2}{c}{ Total } \\
\cline { 2 - 7 } & $\begin{array}{c}\# \\
\text { patients }\end{array}$ & Waiting time & $\begin{array}{c}\text { \# } \\
\text { patients }\end{array}$ & Waiting time & \multicolumn{2}{c}{ \# (and \%) } \\
patients
\end{tabular}

Table 3 gives the average ROT and LOS of all patients (emergent, elective ordinary, and elective day surgery) operated on during the period and their classification with respect to DRG and URG. Note that among the 828 patients operated on during the period (some of them referred in 2009 and others in 2008), 420 have been recognized as DRG 290 (thyroid intervention) as main diagnosis at dismissal. Among the 47 patients coming from Emergent Department, 39 have been operated immediately (URG A), while 8 have a less urgent URG associated meaning that their intervention is postponed and they re-enter as elective patients. The last columns refer to day surgery patients that go home at the end of the intervention day $(\mathrm{LOS}=0)$.

Data related to the service times of the activities/tasks to be performed during the pathway have been also collected. For the thyroid patients the service time of the activities are modeled by triangle distributions using the times collected in Table 1, while for the other patients they have been estimated and the resulting empirical distributions created as model elements. Note that different distribution functions are obtained depending on the urgency class (URG) of patients. For instance, in Figure 4 and 5 the ROT and LOS distributions for the patients belonging to the seven URGs are depicted.

The ROT are split into classes of 30 minutes, from 30 minutes to 7 hours and starting from the number of operations belonging to the same class of each URG the relative frequency is considered, thus deriving the corresponding distribution functions. The LOS are expressed in days and for each URG the frequency distribution function is derived for all patients (emergent and elective), except, of course, the day surgery patients. 
Ozcan, Tànfani, and Testi

Table 3: ROT and LOS of patients operated on during the period analysis

\begin{tabular}{lcccccccc}
\hline & & Emergent & & & & Elective & \multicolumn{2}{c}{ Day Surgery } \\
\hline & $\begin{array}{c}\# \\
\text { patients }\end{array}$ & $\begin{array}{c}\text { ROT } \\
\text { (hours) }\end{array}$ & $\begin{array}{c}\text { LOS } \\
\text { (days) }\end{array}$ & $\begin{array}{c}\# \\
\text { patients }\end{array}$ & $\begin{array}{c}\text { ROT } \\
\text { (hours) }\end{array}$ & $\begin{array}{c}\text { LOS } \\
\text { (days) }\end{array}$ & $\begin{array}{c}\# \\
\text { patients }\end{array}$ & $\begin{array}{c}\text { ROT } \\
\text { (hours) }\end{array}$ \\
\hline DRG & & & & & & & & \\
290 & 1 & 2.05 & 2 & 417 & 2.39 & 4 & 2 & 1.25 \\
others & 46 & 2.16 & 11 & 237 & 2.34 & 8 & 125 & 1.46 \\
& & & & & & & & \\
\hline$U R G$ & & & & & & & & \\
A & 39 & 2.14 & 12 & 11 & 3.08 & 17 & 4 & 2.02 \\
B & 5 & 2.29 & 8 & 71 & 2.52 & 7 & 17 & 1.46 \\
C & 3 & 2.42 & 2 & 285 & 2.28 & 6 & 38 & 1.45 \\
D & 0 & 0 & 0 & 115 & 2.33 & 4 & 31 & 1.36 \\
E & 0 & 0 & 0 & 82 & 2.51 & 5 & 15 & 1.45 \\
F & 0 & 0 & 0 & 53 & 2.44 & 3 & 11 & 1.48 \\
G & 0 & 0 & 0 & 37 & 2.46 & 3 & 12 & 2.19 \\
\hline
\end{tabular}

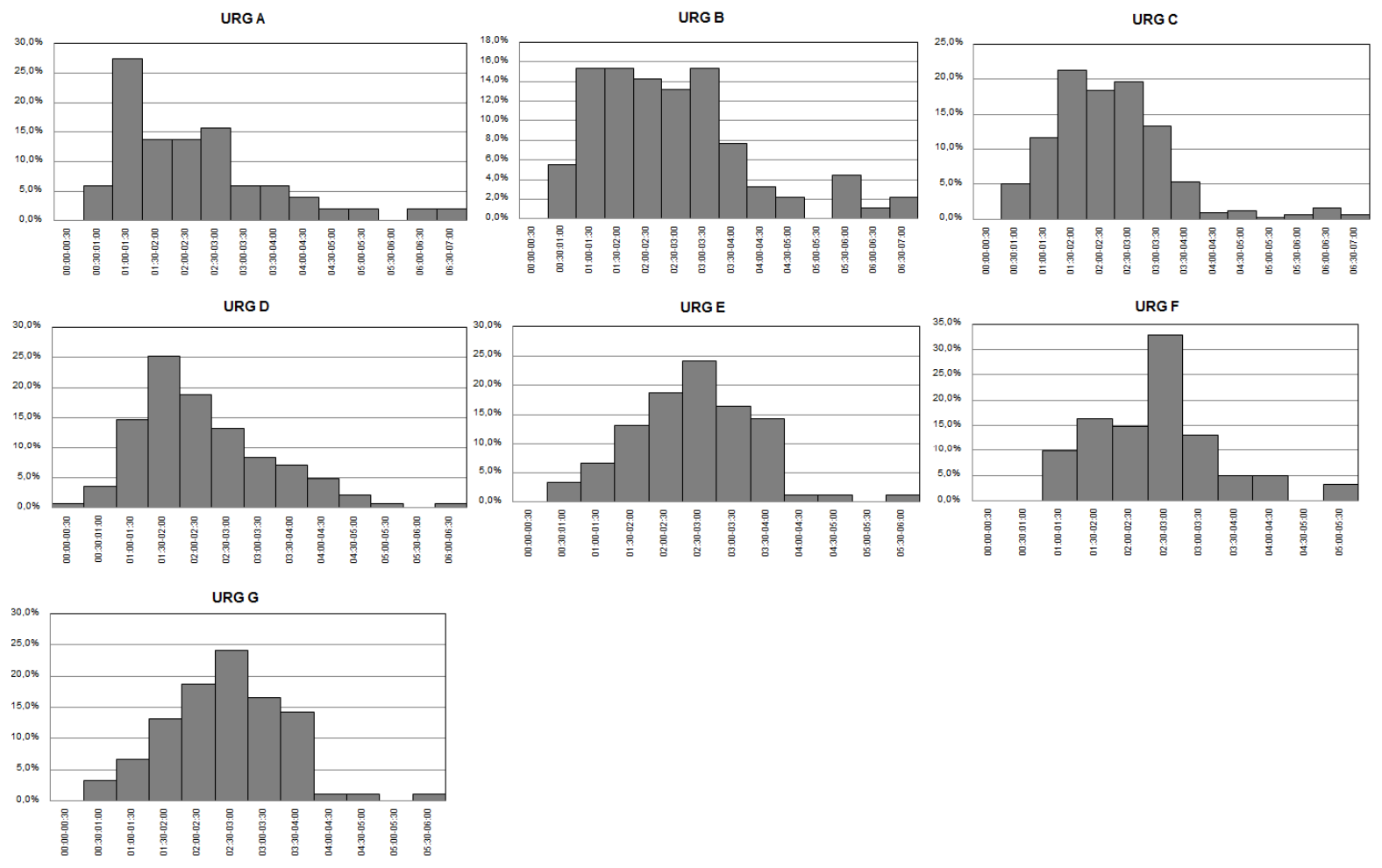

Figure 4: Real Operation Time (ROT) distributions of patients belonging to the different URGs 

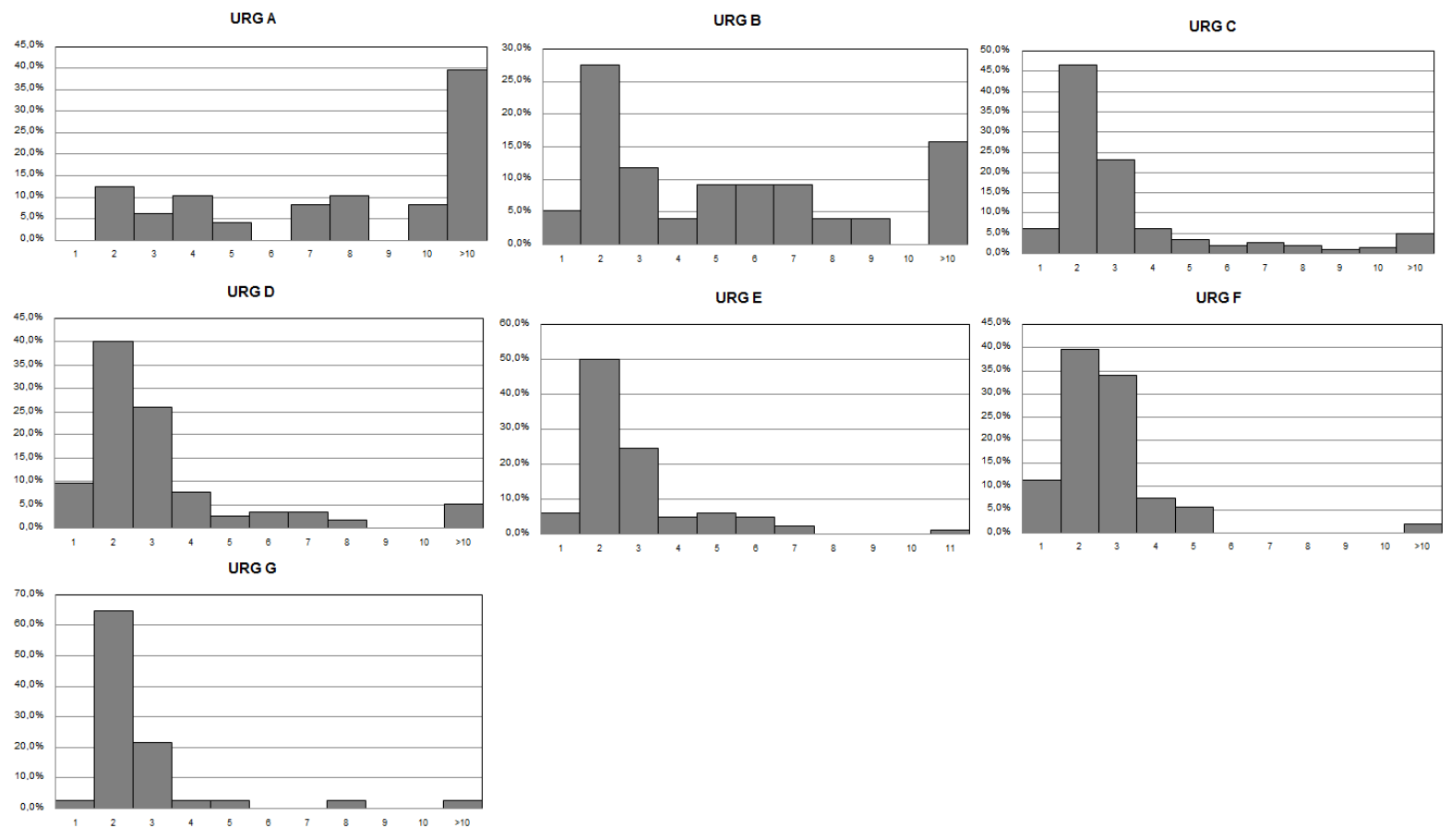

Figure 5: Length of Stay (LOS) distributions of patients belonging to the different URGs

The ongoing activity is aimed at evaluating the inter-arrival distribution functions for all patient types (emergent, elective, thyroid and non-thyroid) and URGs, by applying classical Chi-square and Kolmogorov - Smirnov tests.

The model is already designed to interact with some optimization models at predetermined instants of the simulation run. In particular, at this moment, the interaction is designed on the decisions of how and when ORs are allocated to the specialty under study, referred as Master Surgical Schedule (MSS) problem. In our opinion this is the most critical decision because ORs are the most costly resources affecting all the following sub-process and, thereby, the $\mathrm{CP}$ performance.

To solve the OR planning and scheduling problem, a Minimax optimization model has been already developed to generate optimal OR allocation plans that are used as input parameters for a given period or until some conditions in the system behavior are reached (e.g., the waiting list of some URG patients fall down a given level). When a OR plan revision is needed the optimization model is recalled by the DES model and the simulation output are used as model input data for the next optimization.

\section{EXPECTED RESULTS AND RESEARCH DIRECTIONS}

At present, the main efforts are aimed at verifying the inter-arrival distribution functions of all patient types (emergent, elective, thyroid and not thyroid) and URGs, debugging and tuning the model. Afterwards, particular attention must be given to the validation of the model comparing the model's output with historical data (parametric tests) and verifying the results together with the staff members involved in the data collection (face validity).

We expect to give the first findings of the application of the framework to the thyroid CP during the conference. The main expected results regards the possibility to constrain the resources in various levels and track various performance statistics including: system utilization, queue lengths for patients, patient wait times in queues and total time in the system. The validated model can be used to identify the critical components of the system that represent the process bottlenecks both from a patient-centered (single pathway) and facility-centered (whole system) point of view. The use of simulation allows tracking sys- 
tem variability as well as studying the resource utilization and their impact on the CPs length and waiting time.

Future directions will be devoted to use the framework for performing scenario analyses aimed at evaluating the impact of the organizational changes or alternative management strategies of thyroid clinical pathway and also verifying the robustness of the global health-care system when faced with changes in the epidemiological context like, and in particular, the growth of the incidence of a given pathology which is what is currently happening for thyroid.

\section{ACKNOWLEDGMENTS}

All the authors participate and acknowledge support from the Italian Ministry of Education, University and Research (MIUR), under the grant n. RBFR08IKSB - FIRB PROJECT.

\section{REFERENCES}

Brandeau, M. L., F. Sainford, and W. Pierskalla. 2004. Operations Research and Health Care. A handbook of Methods and applications. Series: International Series on Operations Research \& Management, Vol. 70. Newton: Springer.

Cardoen, B., and E. Demeulemeester. 2008. "Capacity of Clinical Pathways - A Strategic Multi-level Evaluation Tool." Journal of Medical Systems 32(6):443-452.

Cote, M. J., and W. E. Stein. 2007. "A Stochastic Model for Visit to the Doctor's Office." Mathematical and Computer Modeling 45:309-323.

De Blaser, L., R. Depreitere, K. De Waele, K. vanhaecht, J. Vlayen, and W. Sermeus. 2006. "Defining pathways." Journal of Nursing Management 14:553-563.

$\mathrm{Fu}$, M. C. 2002. "Optimization for simulation: Theory vs. practice." INFORMS Journal on Computing 14(3):192-215.

Fu, M. C., F. W. Glover, and J. April. 2005. "Simulation optimization: a review, new developments, and applications. In Proceedings of the 2005 Winter Simulation Conference, edited by M. E. Kuhl, N. M. Steiger, F. B. Armstrong, and J. A. Joines, 83-95. Piscataway, New Jersey: Institute of Electrical and Electronics Engineers, Inc.

Lanner Group. 2010. Witness Manual Guide, Lanner Group.

Kulkarni, R.P., P. H. Ituarte, D. Gunderson, and M. W. Yeh. 2011. "Clinical Pathways Improve Hospital Resource Use in Endocrine Surgery.” Journal of the American College of Surgeons 212(1):35-41.

Maliapen, M., and B. C. Dangerfield. 2010. "A System Dynamics-Based Simulation Study for Managing Clinical Governance and Pathways in a Hospital." Journal of the Operational Research Society 61:255-264.

Ozcan, Y.A. 2009. Quantitative methods in Health Care Management: Techniques and Applications. $2^{\text {nd }}$ ed., San Francisco: Jossey-Bass/Wiley.

Ozcan, Y.A., E. Tànfani, and A. Testi. 2011. "Project Management Approach to Implement Clinical Pathways: An Example for Thyroid Treatment." In Operations Research and Health Care Policy, edited by G. Zaric, in press. Newton: Springer.

Panella, M., S. Marchisio, and F. Stanislao. 2003. "Reducing Clinical Variations with Clinical Pathways: Do Pathways Work?" International Journal for Quality in Health Care 15:509-521.

Ramanujam, L. N., and W. K. Cheah. 2005. "Improvements in Health Care for Patients Undergoing Thyroidectomy." Asia Journal of Surgery 28(4):266-270.

Soria-Aledo, V., B. Flores-Pastor, M. F. Candel-Arenas, A. Carrillo-Alcaraz, A. Campillo-Soto, J. Miguel-Perelló, M. Carrasco Prats, and J. L. Aguayo-Albasini. 2008. "Evaluation and Monitoring of the Clinical Pathway for Thyroidectomy." The American Surgeon 74:29-36. 
Swisher, J., S. Jacobson, J. Jun, and O. Balci. 2001. "Modeling and Analyzing a Physician Clinic Environment Using Discrete Event-Event (Visual) Simulation." Computers \& Operations Research 28:105-125.

Vissers, J., and R. Beech. 2005. Health Operations Management Patient Flow Logistics in Health Care. London: Routledge.

Zander, K. 2002. "Integrated Care Pathways: Eleven International Trends." Journal of Integrated Care Pathways 6: 101-107.

Zander, K., and K. Bower. 1987. Nursing Case Management, Blueprint for Transformation. Boston: Center for Nursing Case Management, New England Medical Center.

\section{AUTHOR BIOGRAPHIES}

YASAR A. OZCAN is Professor in the Department of Health Administration, School of Allied Health Professions at Virginia Commonwealth University. His specialties are mathematical modeling applications in health care, health care information systems, and general statistical applications. His scholarly work is in the areas of health systems productivity, technical efficiency, financial efficiency, and effectiveness for health care providers. More specifically, his specialty is focused on creating benchmarks for physicians and other health care providers using Data Envelopment Analysis (DEA). Dr. Ozcan served as Interim Executive Director, Office of International Education, Virginia Commonwealth University. He is founding Editor-in-Chief of Health Care Management Science and past-president of Health Care Applications Section of Institute for Operations Research and Management Science (INFORMS). His email address is ozcan@vcu.edu.

ELENA TÀNFANI is a Research Assistant in the Department of Economics and Quantitative Methods of the University of Genova, where she teaches "Operations Research for the Management" and "Economics." Her main present research activities are related to the transport and health area, in particular they regard combinatorial optimization models and heuristics methods for planning and scheduling operating rooms, simulation models for the management of waiting lists, performance analysis of hospital department and clinical pathways analysis. Her e-mail address is etanfani@economia.unige.it.

ANGELA TESTI is an Associate Professor in the Department of Economics and Quantitative Methods of the University of Genova, where she holds classes in "Economics," "Advanced Microeconomics," and "Applied Political Economy" at the Economics School; and official courses of "Health Economics" at the School of Medicine, at the School of Law and at the School of Bioengineering. Her main recent research activity regards economic applications in health economics, with particular regards to: i) quantitative analysis of productive processes, health systems productivity, technical efficiency; ii) socioeconomic and deprivation factors affecting health status; iii) equity and prioritization issues. Her e-mail address is testi@economia.unige.it. 